\title{
Issues at the interface between primary and secondary care in the management of common respiratory disease
}

\section{Introduction}

\author{
W F Holmes, John Macfarlane
}

This commentary introduces a new series for Thorax which, over the next six issues, will consider some of the common ground between respiratory physicians and primary care physicians (general practitioners (GPs) in the United Kingdom). Respiratory illnesses are among the most common reason for consultations in general practice and for acute hospital admissions, and much of the follow up of important respiratory disease is provided by general practitioners.

General practitioners often complain, and not without some justification, that partnership with secondary care may be a euphemism for inappropriate, poorly communicated, and inadequately resourced transfer of responsibility at hospital discharge, or a means of using them to collect research data for their hospital colleagues. Respiratory physicians, however, can be proud of their record of collaboration with general practitioners. In the management of asthma in particular, primary care has been actively involved in a new understanding of the pathogenesis of asthma and, by working together, respiratory physicians, general practitioners, and nurses have achieved a fundamental change in the management of a common disorder. The British Thoracic Society's guidelines on the management of asthma ${ }^{12}$ were not the first to be published but were widely welcomed and enjoy a continuing respect which guidelines from few other disciplines have matched. Initiatives in other respiratory areas such as chronic obstructive pulmonary disease (COPD) ${ }^{3}$ have sought a seamless management plan between primary and secondary care.

Most general practitioners have shown themselves ready to respond to this leadership and willing to accept the invitation to participate. Some consequences of this collaboration are clear, particularly an appropriate modification of prescribing to reflect the need for inhaled medication and anti-inflammatory therapy. Nonetheless, considerable problems remain which have proved less easy to address. Communication between professionals following hospital discharge continues to show considerable room for improvement, and primary care may be less good at organising research and audit, particularly between rather than within practices, a problem which will be a major challenge for primary care groups.

There has been a change, too, in philosophy. We increasingly recognise that primary care is the most suitable location for management and follow up of much respiratory disease, not because of scarce resources (bluntly, "it would be best to be followed by a hospital expert but we don't have enough of them") but rather because of a change in thinking ("primary care is actually the best place for this condition to be supervised"). Individual units may provide an excellent service, yet, although we may have some agreement over what constitutes quality care, we have less idea of how it can be delivered universally.

Even deceptively simple questions pose major challenges. What is "appropriate" follow up of a chronic illness? We have some consensus but not much evidence to decide. What is the role of expert advice? Is it effective at improving the behaviour of those to whom it is directed, both patients and their general practitioners? Evidence based upon a demonstrable effect on outcome, rather than on perceived best practice, is more likely to generate appropriate advice.

Formulating relevant questions and gathering the evidence to answer them is best achieved with the active participation of primary care, but this is no simple task as collaboration between disciplines is never easy. There are traditions to be respected as well as organisational problems to surmount. Every day general practitioners cope skilfully with uncertainty in the diagnosis and management of acute self limiting illness. However, a strategy for symptomatic conditions- "do this and come back and see me if it doesn't get better"-although undoubtedly efficient in experienced hands, tends to fall down in the management of chronic diseases such as COPD or in palliative care where patients either tolerate symptoms or continue to receive medication which is unhelpful.

This series of six articles will address a number of common problems which cross the interface between primary and secondary care: lung cancer, pneumonia, antibiotic prescribing for acute respiratory illness, asthma in children and adults, and COPD. Each article has been jointly written by a respiratory physician and a 
general practitioner (and, in the case of lung cancer, with a palliative medicine physician). The articles are not tutorials in current management; rather, they offer a personal review of some of the clinical problems which exist in that field and some of the organisational difficulties to be overcome. They will also pose a number of questions which could be usefully addressed at a time when there is both enthusiasm and funds to pursue important research in primary care. We hope they will stimulate discussion and perhaps focus attention on many areas ripe for further investigation and support.

Improving the overall management of these respiratory conditions is unlikely to be achieved by either discipline alone, but rather by combining the specialist expertise and investigations available in secondary care with the continuity which primary care ought to provide. ${ }^{4}$ The links between respiratory physicians and general practitioners are already strong. Drawing these two resources and traditions further together should, if done properly, be to the advantage of patients, doctors, and the institutions in which they work.

1 Statement by the BTS and others. Guidelines for the management of asthma in adults: 1. Chronic persistent asthma. BMF 1990;301:651-3.

2 Statement by the BTS and others. Guidelines for the management of asthma in adults: 2 . Acute severe asthma. BMF 1990;301:797-800.

3 COPD Guidelines Group of the Standards of Care Committee of the British Thoracic Society. BTS guidelines for the management of chronic obstructive pulmonary disease. Thorax 1997;52(Suppl 5):S1-28.

4 Pereira Gray D. Forty seven minutes a year for the patient. Br f Gen Pract 1998;48:1816-7. 